\title{
On the Implementation of GNU Prolog
}

\author{
DANIEL DIAZ \\ University of Paris 1 \\ 90 rue de Tolbiac \\ 75013 Paris, FRANCE \\ E-mail: Daniel.Diaz@univ-paris1.fr \\ SALVADOR ABREU \\ Universidade de Évora and CENTRIA FCT/UNL \\ Largo dos Colegiais 2 \\ 7004-516 Évora, PORTUGAL \\ E-mail: spa@di.uevora.pt \\ PHILIPPE CODOGNET \\ JFLI, CNRS / University of Tokyo \\ $J A P A N$ \\ E-mail: Philippe.Codognet@lip6.fr \\ submitted 4th October 2009; revised 1st March 2010; accepted 22nd November 2010
}

\begin{abstract}
To appear in Theory and Practice of Logic Programming (TPLP).

GNU Prolog is a general-purpose implementation of the Prolog language, which distinguishes itself from most other systems by being, above all else, a native-code compiler which produces standalone executables which don't rely on any byte-code emulator or meta-interpreter. Other aspects which stand out include the explicit organization of the Prolog system as a multipass compiler, where intermediate representations are materialized, in Unix compiler tradition. GNU Prolog also includes an extensible and highperformance finite domain constraint solver, integrated with the Prolog language but implemented using independent lower-level mechanisms. This article discusses the main issues involved in designing and implementing GNU Prolog: requirements, system organization, performance and portability issues as well as its position with respect to other Prolog system implementations and the ISO standardization initiative.
\end{abstract}

KEYWORDS: Prolog, logic programming system, GNU, ISO, WAM, native code compilation, Finite Domain constraints

\section{Introduction}

GNU Prolog's roots go back to the start of the 1990s at the Logic Programming research team at INRIA Rocquencourt, in Paris. Philippe Codognet planned on implementing a low-level version of his intelligent backtracking techniques and opted to do so on the state-of-the art SICStus Prolog system, for which he obtained the source code. This task was handed down to Daniel Diaz, at that time an M.Sc. student. However, at the time SICStus Prolog was already a very large scale and 
complex system: version 2.1 had about 70,000 lines of highly tuned and optimized $\mathrm{C}$ and Prolog code: clearly not the easiest platform on which to carry out independent, low-level experiments. So we took it upon ourselves to develop yet another implementation of Prolog which would meet the following requirements:

- The system ought to serve as the basis for several research-oriented extensions such as: intelligent backtracking, co-routining (freeze was a hot topic back then), concurrency, constraints, etc.

- The system would be made available freely to all researchers.

- The system would be portable by design, not tied to any particular architecture.

- The core of the system must be simple and lightweight, unlike SICStus.

- The base system performance should be good. The rationale being that a system designed to be extended needs to provide good base performance. We targeted performance close to that of SICStus Prolog native code.

The last two points (simplicity and performance) were hard to reconcile. This was particularly true at a time where research on WAM optimization was a hot topic: choice points, backtracking, unification, indexing, register allocation, etc.: all aspects of the WAM were the object of published research on optimizations thereof. The goals we had set for ourselves seemed difficult to reach and rather ambitious: performance with a simple implementation, all done with little or no optimizations.

At the time, most Prolog systems were based on byte-code emulators, written in $\mathrm{C}$ or assembly language. We decided we would need to compile to native code in order to recoup the relative performance loss due to the inherent simplicity of the WAM model we were to adopt. It remained to be seen how we would go about producing native code. At the time, producing native code seemed to be the best thing one could possibly do: see BIM-Prolog, SICStus Prolog, Aquarius Prolog, to name a few. The SICStus approach was to retain its usual emulated byte-code and only present the option of producing native code for select architectures 1 , which could be transparently mixed with emulated predicates. The approach followed by Peter van Roy with Aquarius Prolog was different and more traditional: compilation was separate from execution, as in regular programming languages (Van Roy and Despain 1992). A "command line" compiler would translate a Prolog program into a nativecode executable. Unfortunately, simplicity was apparently not a goal of the exercise: Aquarius' abstract machine, the BAM, was lower-level and finer-grained than the WAM and comprised more than 100 instructions, some of which were specializations created on-the-fly by the compiler using abstract interpretation techniques. Other "native code generation" approaches were surfacing, which would generate $\mathrm{C}$ rather than an actual assembly language: these included Janus (Gudeman et al. 1992) which translated Prolog to C, KL/1 (Chikayama et al. 1994) which compiled a language different from Prolog (a committed-choice language, with don't-care nondeterminism) or Erlang (Hausman 1993), a functional language only loosely related to Prolog. A significant difficulty that compilers to $\mathrm{C}$ had to deal with was the

\footnotetext{
${ }^{1}$ Sparc under SunOS was the chosen one.
} 
orthogonal control dimension due to backtracking, which complicates stack frame management beyond what $\mathrm{C}$ can normally do.

Our choice was to translate to $\mathrm{C}$ via the WAM, decorating the generated code with direct assembly language instructions, to handle native jumps which correspond to Prolog control transfers, such as the call, execute or proceed instructions. The system we built was called wamcc (Codognet and Diaz 1995) and most WAM instructions were directly replaced by the equivalent $\mathrm{C}$ code, inlined at compile-time via a set of $\mathrm{C}$ Preprocessor macros. More complex instructions result in library function calls, this was the case with unification instructions, for example. Lastly, wamcc was the first documented Prolog system to rely on the hardware (MMU) to detect stack or heap overflows, by placing unmapped pages at the limits of the dynamic memory areas: accessing these would raise an exception and interrupt the normal flow of execution. This approach resulted in a clear performance gain when compared with bounds checks being performed on every allocation and was innovative at the time ${ }^{2}$ Our choices clearly paid off, as wamcc got a $60 \%$ performance gain w.r.t. emulated SICStus and about $30 \%$ performance loss w.r.t. SICStus native, the agreed-upon references of the time.

Since then, wamcc has been used as a teaching tool in several universities and, outside INRIA, as the starting point for research work, e.g. (Ferreira and Damas 1999). The next step ought to have been the implementation of intelligent backtracking in wamcc. This was not to happen: our interest shifted to the blooming area of Constraint Logic Programming (Jaffar and Lassez 1987), which was suddenly and for the first time enabling Logic Programming for large-scale industrial applications. There is little doubt that Pascal Van Hentenryck's PhD thesis work and the implementation of a Finite Domain (FD) solver in the CHIP language (Van Hentenryck 1989) were instrumental in CLP's success. The CLP mechanisms underlying CHIP were touted as highly optimized, totalling $50 \mathrm{~K}$ lines of $\mathrm{C}$ code and shrouded in wraps of secrecy - a very useful and interesting black box. It took a few years for the work describing cc(FD) (Van Hentenryck et al. 1994) to provide hints as to how an effective CLP system could be implemented. The following move for wamcc was clear: it would become clp(FD) and introduce a simple extension to the WAM to integrate FD constraints (Diaz and Codognet 1993, Codognet and Diaz 1996). clp(FD) was about 4 times faster than CHIP.

The worst problem we had with wamcc (and consequently clp(FD)) was the excessively long time it took GCC to compile the generated C code. Even for moderatelysized programs, the time quickly became overwhelming and even our attempts to banish most inlining in favour of library function calls were insufficient to bring the times down to acceptable levels.

On closer inspection, the $\mathrm{C}$ language was being used as a machine-independent assembly language, which we had to fool in order to do jumps. This went against the regular operation of a $\mathrm{C}$ compiler, which expects regular function entry and exit to be the norm: as a consequence the compiler was trying to do its task over programs

2 These days, this functionality is available off-the-shelf, in the form of the libsigsegv library, on http://libsigsegv. sourceforge.net/ 
which were really too devious and too large for it to properly cope. As we did not really need all that $\mathrm{C}$ could express, we started looking for alternative languages to compile into. One possibility which looked very interesting was C-- (Jones et al. 1997). Unfortunately this system was not developed to the point where it would be actually useful for our purpose: it remains bound to a limited set of back-ends ${ }^{3}$ We then set out to specify and implement an intermediate language of our own. Our "Mini-Assembly" (MA) language would have to meet very basic requirements: to directly handle WAM control and be able to call $\mathrm{C}$ functions would be sufficient. This simplicity was meant to promote the easy porting to common architectures.

At that time, the Prolog standardization effort was in full swing, which lead to the emergence of the specification document known as ISO Core 1 (ISO-Part1 1995). We then committed to develop a completely new, standard-compliant implementation of Prolog, which would use the MA intermediate language instead of $\mathrm{C}$ in the compiler pipeline. So was GNU Prolog born, under the code name Calypso.

The Prolog language was not very popular (euphemism alert!) outside the research community, and in particular no implementation of the language was present in the GNU organization catalogue, whereas other, similarly exotic languages such as Scheme, were. We took it upon ourselves to defend our case with GNU in late 1998 and the first official release of GNU Prolog saw the light of day in April 1999. GNU Prolog is presently directly available for most Linux distributions.

The remainder of this article is structured as follows: Section 2 discusses the structure of the GNU Prolog compiler pipeline with fully fleshed-out examples. In Section 3 we present GNU Prolog's Constraint Logic Programming design and implementation. Section 4 tackles the positioning of GNU Prolog in the Prolog landscape and its relation to the ISO standardization initiative. Finally, in Section 5 we draw conclusions from the experience acquired over the last years and lay out possible plans for further developments, some of which are actively being pursued by the authors of the present article as well as other researchers.

\section{Compilation Scheme}

In this section we detail the compilation scheme adopted in GNU Prolog. As previously stated, the main design decision was to use a simple WAM and to compensate for the lack of optimizations by producing native code, thereby avoiding the overhead of an emulator. The compilation process is then the key point of GNU Prolog. In wamcc, GNU Prolog's ancestor, we produced native code via C: a Prolog file was translated into a WAM file, itself translated to $\mathrm{C}$ and finally to object code, by the $\mathrm{C}$ compiler. This approach had a major drawback: the time needed to compile the $\mathrm{C}$ file; even for medium-sized Prolog sources, the time needed by the $\mathrm{C}$ compiler was quickly dominating the entire compilation process. In GNU Prolog we decided to sidestep the issue by not compiling to $\mathrm{C}$ but, instead, to directly generate assembly code. The direct translation from the WAM to assembly code turns out to be a

\footnotetext{
${ }^{3}$ For a long time C-- was restricted to 32 -bit x86, and even though this situation has evolved, the set of target architectures is still smaller than what we have attained with the MA tool.
} 
significant effort as a translator must be written for each target architecture. We simplified this problem by defining an intermediate language called Mini-Assembly or MA for short, which can be viewed as a machine-independent assembly language well suited to be the target of a WAM translator. This language will be detailed below, in sections 2.2 and 2.3 .

One of the design goals for GNU Prolog was to offer a system which can easily be extended by other research teams. This requirement led us to split the compiler into several passes, with distinct executables for which the respective intermediate representations must be materialized as plain text files or streams. The passes are:

pl2wam which compiles a Prolog source file into WAM code.

wam2ma which converts the WAM code to the MA language.

ma2asm that translates the abstract MA code to architecture-specific machine instructions: its output is an assembly language program.

fd2c compiles FD constraint definitions into $\mathrm{C}$ functions which perform constraint propagation at runtime. See section 3 for more on this.

In addition to those GNU Prolog-specific compiler components, the compilation process also involves the standard tools:

as the assembler for the target architecture.

cc the $\mathrm{C}$ compiler

Id the link editor: to bind together all objects/libraries and provide a machinedependent executable.

All of these are depicted in Figure 1 . The general compiler driver, called gplc, manages all appropriate passes and intermediate files, according to the provided input files and the desired output. For instance, the user can mix input file types (Prolog, WAM, MA, object files, libraries, etc.) as well as ask the compiler to stop after any intermediate stage. The type of a file is determined using the suffix of its file name and is used to select its processor in the toolchain.

\subsection{Compiling Prolog to WAM}

Compiling Prolog to WAM is a well known and documented subject. GNU Prolog is based on the original WAM (Warren 1983, Ait-Kaci 1991) but uses a simple one-level indexing mechanism instead. As previously mentioned, the WAM variant we are compiling to is not very sophisticated, for instance the code for structure unification does not handle read and write modes separately, shallow backtracking is not implemented, there is no separate choice stack (choice-points reside in the local stack), to name but a few known techniques which are not used, for the sake of simplicity. Nevertheless, a few simple optimizations did get implemented, namely: improved register allocation, unification instruction reordering, inlining for some built-in predicates, last call and last subterm (Carlsson 1990) optimizations.

It is possible to disable any or all of these optimizations using gplc command line flags, a possibility which is particularly interesting for people wishing to become 
source file which applies to the subsequent predicate definitions. Note that several instances of file_name/1 may occur, as a result of include/1 directives. The fact for predicate/ 6 contains the code for the predicate conc/3, as a list of WAM instructions. Several predicate properties are also stated here: static (as opposed to dynamic), private (as opposed to public) and user (as opposed to built-in).

This code can be easily read and understood by humans (useful for student use) and it can also directly serve as input for a Prolog program like an emulator, a source-to-source optimizer or another back-end as was done in the Prolog-to-EAM compiler, reported on in (André and Abreu 2010). The drawbacks of this choice are: a not very compact representation (see the length of the instruction names, for instance) and the need for a non-trivial parser for the next stage which, in the case of GNU Prolog, is handled by wam2ma.

As previously mentioned, the GNU Prolog WAM is not very optimized. Consider the clause: $p(a, X):-q(a, X), r(X)$. Compiling it results in the following suboptimal code (2 WAM instructions could be avoided):

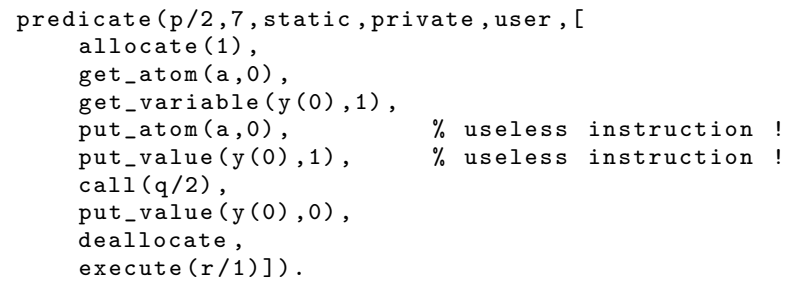

A WAM instruction cache could solve this as explained in (Carlsson 1990). Basically, the cache remembers the current values of the WAM registers. When a put instruction occurs, if the cache detects the wanted data is already present in a register it replaces the put instruction by a register move instruction (hoping the register optimizer will delete this move intruction). Optimizations such as this are not included in our current compiler, which remains simple-about 3000 lines of Prolog code-yet adequately efficient. Consider file bool.pl and the corresponding bool. wam shown in figure 3 the generated WAM code is, in this case, close to optimal. Observe how cut is handled: with the first instruction (line 4), the cut level is stored in WAM temporary register X(1) Afterwards, it is treated as any other Prolog variable: it only gets copied into a permanent variable- $\mathrm{Y}(0)$ at label (6)-because its value is needed after the first chunk, for the cut.

In short: pl2wam is a simple and portable Prolog-to-WAM compiler, written in Prolog, which produces text files with a representation of WAM programs. The quality of the generated code, while not outstanding, is adequate for our purpose.

\subsection{From the WAM to Mini-Assembly}

The next stage in the GNU Prolog compiler pipeline translates WAM instructions to our MA intermediate language. This language has been designed specifically for

4 In fact, this would actually be the first available X register, i.e. X (arity) since we count from zero. This technique is similar to that used in XSB (Rao et al. 1997) or SICStus (Carlsson 1990). 
- It handles the control of Prolog well: WAM instructions like call, execute, return and fail result in native jumps.

- It can call a $\mathrm{C}$ function with a wide variety of arguments and can use the return value in several ways:

- To store it in memory or in a WAM register.

- To test its value and, to fail if zero (like fail).

- To branch to the address specified by the return value (e.g. the address returned by switch_on_term.)

- It has a small instruction set (to facilitate the mapping to concrete machines) and only knows about a subset of the WAM, mainly that which is necessary for execution control 5

- It is possible to declare scalar variables and arrays (only of type long).

- It is possible to declare initializer code, which will be automatically executed at run-time. This issue is further discussed in section 2.3 .

The next section discusses and details the MA Instruction Set Architecture.

\subsection{The MA Instruction Set}

Here is a description of each MA instruction:

$\mathrm{p} l_{-}$jump $p l_{-} \_a b e l$ : continue execution at the predicate whose symbol is $p l_{-} l a b e l$. This symbol is an identifier whose construction is explained in later on. This corresponds to the WAM instruction execute.

pl_call $p l_{-} l a b e l$ : continue execution at the predicate whose symbol is $p l_{-} l a b e l$, after having set the continuation register CP to the address of the very next instruction. This corresponds to the WAM instruction call.

pl_ret: continue execution at the address given by the continuation pointer CP. This corresponds to the WAM instruction proceed.

pl_fail: continue execution at the address given by the last alternative, i.e. the ALT cell of the last choice point, itself given by the WAM register B. This corresponds to the WAM instruction fail.

jump label: continue execution at the symbol label, which can be any (predicate local) label. This instruction is used when translating WAM indexing instructions (e.g. try, retry or trust) to perform local control transfer, i.e. branching within the same predicate. This specialized version of $\mathrm{pl}_{-}$jump exists because, in some architectures, local jumps can be optimized.

call_c $f_{-}$name $(\arg , \ldots)$ : call the $\mathrm{C}$ function $f_{-}$name with arguments $\arg , \ldots$ Each argument can be an integer, a floating point number (C double), a string, the address of a label, the address or contents of a memory location, the address or contents of a WAM X register or Y permanent variable. This instruction is used to translate most WAM instructions. The return value of the function call can only be accessed by one of the *_ret instructions which follow.

${ }^{5}$ For instance, the fail instruction needs to know about the B register and a displacement from it to get the alternative address to backtrack to. 
fail_ret: perform a Prolog fail (like pl_fail) only if the value returned by the preceding $\mathrm{C}$ function call is 0 . This instruction is used after a $\mathrm{C}$ function call returning a boolean to indicate its outcome, typically unification success.

jump_ret: continue execution at the address returned by the preceding $\mathrm{C}$ function call. This instruction makes it possible to use $\mathrm{C}$ functions to determine where to transfer control to. For instance, the WAM indexing instruction switch_on_term is implemented by a $\mathrm{C}$ function returning the address of the selected code.

move_ret target: copy the value returned by the previous $\mathrm{C}$ function call into target which can be either a memory location or a WAM X or Y variable.

c_ret: C return. This instruction is used at the end of the initialization function (see below) to give the control back to the caller.

move reg1, reg2: copy the contents of the WAM X or Y variable reg1 into reg2.

The extreme simplicity of the MA language is noteworthy. Observe, however, the presence of the move instruction which performs a copy operation on WAM X registers or on permanent variables: while not strictly necessary ${ }^{6}$ moves between variables are very frequent and the invocation of a $\mathrm{C}$ function would be costly in terms of execution time. This reflects a tradeoff between the minimality of the instruction set and acceptable performance. It would be possible to extend the instruction set (e.g. adding arithmetic instructions) but doing so would complicate writing the architecture-specific back-ends with little expected gain.

In addition to the above instructions, MA also supports declaration statements. In what follows, the keyword local is used for a local symbol (only visible within the current object file) while global allows others to see that symbol.

pl_code global $p l_{-} l a b e l$ : define a Prolog predicate with name $p l_{-} l a b e l$. At present all predicates are tagged global (i.e. visible by all other Prolog objects), but local will be used when implementing a module system.

c_code local/global/initializer label: define a function that can be called from C. The use of initializer ensures that this function will be executed early, before the the Prolog engine is started. At most one initializer function may be declared per file.

long local/global $i d=$ value: allocate the space for a long variable whose name is $i d$ and initialize it with the integer value. The initialization is optional.

long local/global id(Size): allocate the space for an array of Size longs whose name is $i d$.

The WAM to MA translation done by wam2ma is performed in linear time w.r.t. the size of the WAM file (the translation is performed on the fly as the WAM file is being read). This is the behaviour that we sought in generating MA rather than C.

Like with the pl2wam phase, the result of wam2ma is a text file that can be easily used as input for another program.

We now present the MA code obtained for our bool.pl example (we have added the corresponding WAM instruction as comments):

${ }^{6}$ Instead, we could easily invoke a C function to copy the data, using the call_c instruction and an extra library function. 


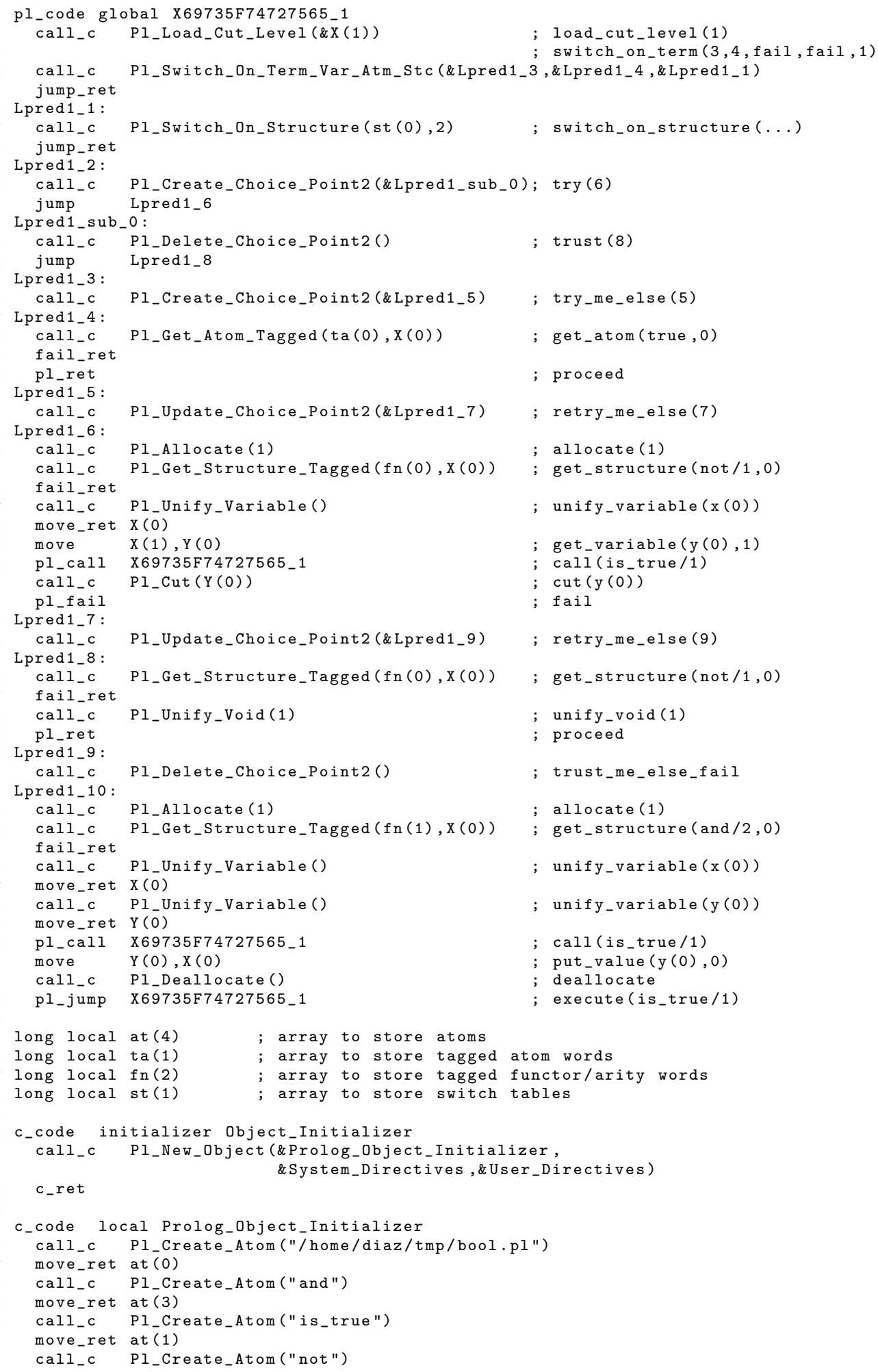




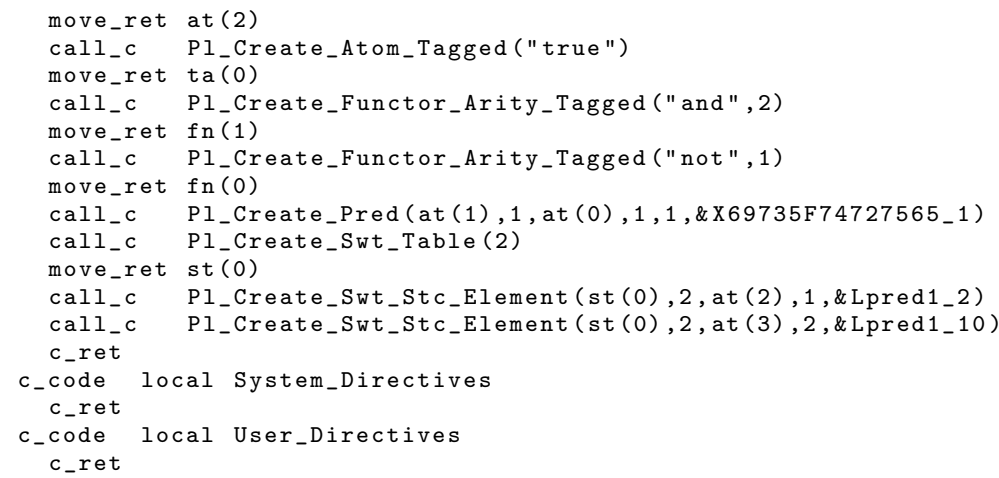

From this example, one can observe that most WAM instructions map to a $\mathrm{C}$ function call, following the subroutine-threading pattern. As previously mentioned, control instructions are directly translated to their corresponding MA counterparts. Another exception concerns moves between WAM registers and permanent variables (e.g. lines 29 and 51). After further analysis of this example, several remarks can be made:

Line 1: (predicate label, load_cut_level...) this is the start of the code associated to predicate is_true/1. As the MA language is to be mapped to the assembly language of an actual target machine, we decided that only "classical" identifiers can be used (a letter followed by letters, digits or the underscore character). In particular, it is necessary to associate such an identifier to each predicate (referenced as $p l_{-} l a b e l$ in Section 2.3.

Since the syntax of assembly identifiers is more restrictive than the syntax of Prolog atoms (which may include any character using quotes) GNU Prolog uses a normalized hexadecimal-based representation for identifiers, where each predicate name is translated into a symbol beginning with an $\mathrm{x}$, followed by the hexadecimal notation of the code of each character in the name, followed by an underscore and the arity. For example, predicate symbol is_true/1 is encoded as the symbol X69735F74727565_1 (69 is the hexadecimal representation of "i", 73 of "s", and so on).

The linker is responsible for resolving external references (e.g. calls to built-in or user predicates defined in another object). The output of the linker is filtered by GNU Prolog to descramble hexadecimal symbol encodings, in case there are errors (e.g. an undefined predicate, multiple definitions for a predicate).

Line 4: (switch_on_term...) the switch_on_term WAM instruction maps to a $\mathrm{C}$ call to a specialized function P1_Switch_On_Term_Var_Atm_Stc which takes only three arguments (it checks if the first argument is a variable, an atom or a structure). This is more efficient than calling the general $\mathrm{C}$ function with all 5 arguments (integer and list) as the call is cheaper (fewer arguments are passed) and faster (fewer cases to test).

Line 7: a switch_on_structure in the WAM code results in the creation of a switch table (done in the initializer part). At execution time, this table is passed to the $\mathrm{C}$ function (the other argument is the size of the table - in this case, two elements: not/1, and/2). A hash-table with only two elements is not very efficient, a nested if would be better: clearly, there is room for improvement.

Lines 10-16: functions handling choice points are also specialized (here the functions are for a choice point with two arguments: the first argument in $\mathrm{X}(0)$ and the cut-level in $\mathrm{X}(1)$. Such specialized functions exist for arity $<4$. For greater arities, the arity must be passed as a parameter to the generic function.

Line 18: the get_atom WAM instruction maps to a $\mathrm{C}$ function call to P1_Get_Atom_Tagged. 
This function takes as first argument a tagged atom, i.e. a WAM word. This value is created by the initializer function and stored in module-local array ta at index zero $7^{7}$ Doing so avoids having to call the tag/untag function at runtime. Here it is used to dereference $\mathrm{X}(0)$ and unify its value with ta(0).

The same occurs on lines 25, 36 and 44 where the get_structure instructions get mapped to calls to P1_Get_Structure_Tagged which takes a single-word encoding of the functor and arity. These are created by the initializer and stored in a module-local array $\mathrm{fn}(\ldots)$.

Lines 55-58: several arrays are declared to store atoms, tagged atoms, tagged functor/arity and switch tables.

Line 60: the initializer is declared. It simply calls a $\mathrm{C}$ function to register this new object (an object file generated by the compilation process). It passes 3 function pointers:

- Prolog_Object_Initializer: the initializer function for the object. This function creates the atoms, the switch tables, the tagged words, etc.

- System_Directives: executes system directives, such as op/3 or char_conversion/2.

- User_Directives: is the entry point for the procedure which executes the user startup code (defined with Prolog initialization directives).

It is worth noting that the code needed to install the object (i.e. the code in the body of Prolog_Object_Initializer) cannot be directly executed in the initializer (i.e. in Object_Initializer) because that code is executed very early: when the OS loads and runs the executable, i.e. before the main function is called. At this time, the required global Prolog data structures (e.g. atom hash table) are not yet allocated. We therefore limit ourselves to registering the object and, only when all Prolog data areas are ready do the Prolog initializer functions get executed.

Line 64-84: the object initialization function creates the objects required by the code: atoms, tagged atoms, tagged functor/arity words, switch tables and stores these in the object's local arrays. Atoms are classically hashed and thus can only be known at runtime (since we can have multiple files linked together). The initializer registers all needed atoms and stores them in local variables (e.g. in the ta array). Notice that this could be optimized since once this information is created it remains constant during the execution of the program. One could imagine a 2-pass optimizer: only execute all initialization functions to detect the values of all involved atoms, then recompile the whole using integer constants instead of MA array variables. The impact of this optimization would be very important if atoms are very used since it is much faster to pass an integer than loading it from the memory. Another benefit of this optimization would be the reduction of the startup time in applications which have a large number of atoms. Finally, note that the initializer also registers the predicate is_true/1 with the P1_Create_Pred function (line 79): this is only needed for meta-calls to work, because we need to associate an address (given here by the linker-resolved symbol X69735F74727565_1) to the predicate symbol. Other arguments are the file name and the line number where it is defined, and a mask containing the properties of the predicate.

To summarize, the Mini-Assembly language has a few control-flow instructions, pseudo-ops to control constants and data areas, the $\mathrm{C}$ function invocation operation and register movement instructions. Predicate names are hashed into linker-friendly names. These features make it sufficient as the target for compiling WAM code.

7 ta stands for "tagged atom". 


\subsection{From Mini-Assembly to Actual Assembly}

The next stage consists of mapping the MA language generated in the previous section to the target machine's actual instructions. Since MA is based on a very small instruction set, the writing of such a translator is inherently simple. However, producing machine instructions is not an easy task. The first MA-to-assembly language mapper was written with the help of snippets taken from a $\mathrm{C}$ file produced by wamcc: indeed, compiling a Prolog file to assembly by means of gcc gave us a starting point for the translation, as the MA instructions correspond to a subset of the $\mathrm{C}$ code. We then generalized this approach by defining a $\mathrm{C}$ file, each portion of which corresponds to an MA instruction: the study of the assembly code produced by gcc was our reference. This provided preliminary information about register use conventions, C calling conventions, etc. However, in order to complete the assembly code generator, we need to refer to the technical documentation of the processor together with the ABI (Application Binary Interface) used by the operating system.

We now show portions of the assembly code for the previous example, using the linux/i86_64 target 8 We focus on the code for the last clause of is_true/1, annotated with the corresponding WAM \& MA code:

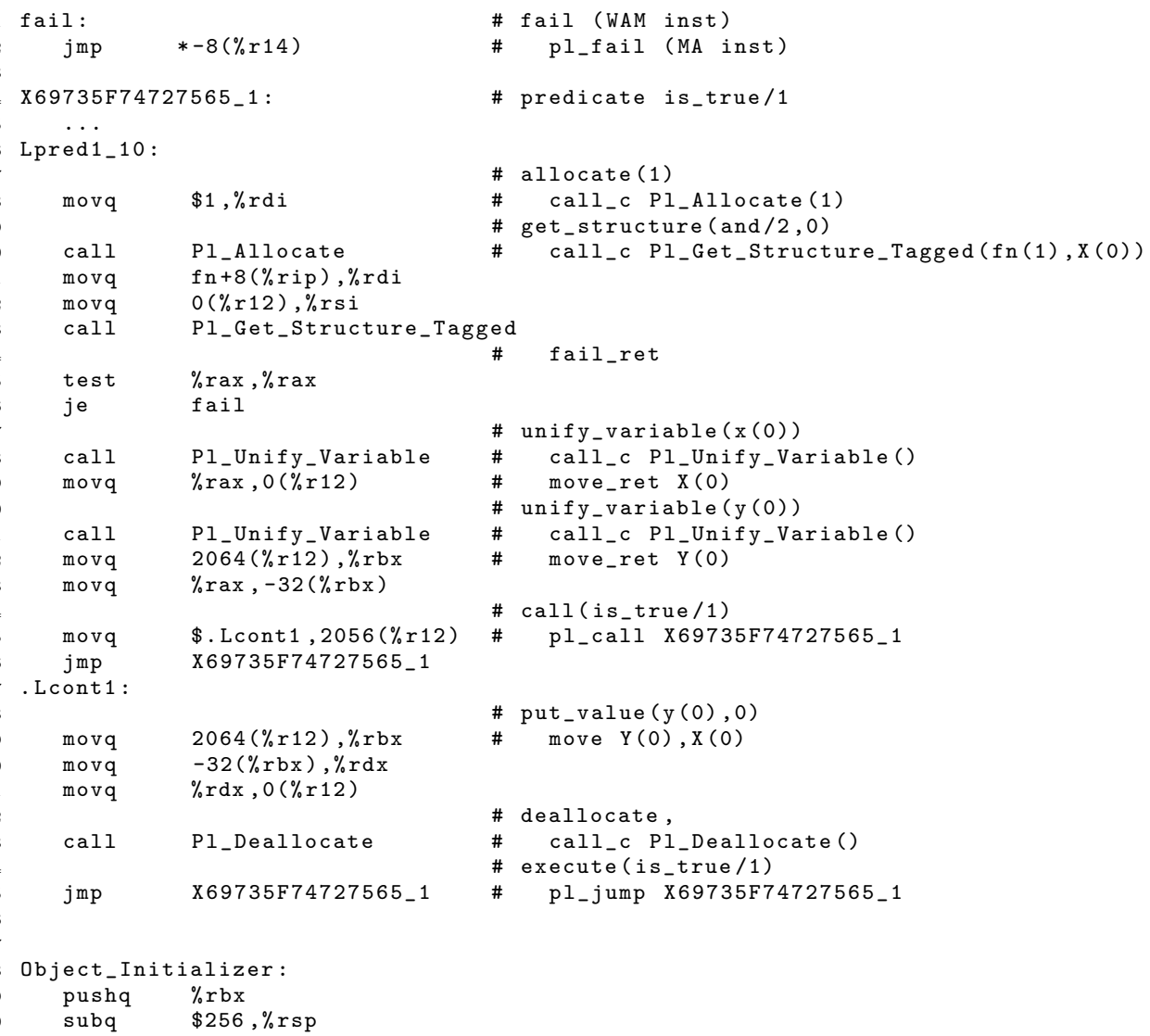

8 We are still using the same example, meaning this is file bool.s. 


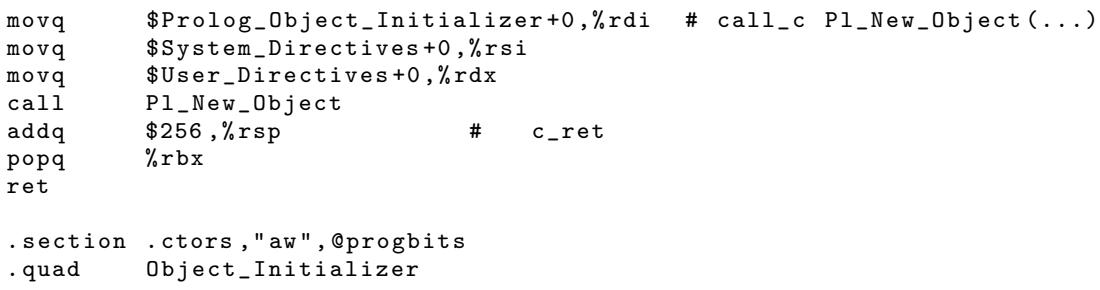

A few observations:

Line 1: a label is defined to perform a WAM fail. Each time a fail is needed, a jump is performed to this label (e.g. Line 16 for the MA instruction fail_ret). We can see that the last choice point frame (B) is stored in the x86_64 register \%r14 and the alternative (ALTB) is the first 64 bits cell just below the address pointed by B. An indirect jump does the work.

Line 12-13: a call to a $\mathrm{C}$ function yields an assembly call instruction, respecting the x86_64 ABI: the arguments are passed via the registers \%rdi, \%rsi, \%rdx,... Line 12 also reveals that the address of the bank of WAM temporaries ( $\mathrm{X}(\ldots)$ variables) is kept in the register \%r12. Other used registers are \%r13 for the top of the trail (TR) and \%r15 for the top of the heap $(\mathrm{H})$.

Lines 25-26: a WAM call instruction gives produces 2 assembly instructions. The first one stores the next address (a local label) in the CP register (accessed as an offset from $\%$ r12). The second instruction simply jumps to the called predicate.

Lines 38-47: the initializer which calls a $\mathrm{C}$ function to register this object.

Lines 49-50: fill the CTORS sections ("constructors") with a new entry: Object_Initializer.

At run-time, the contents of this section is interpreted as an array of addresses, all of which are executed as functions (see section 2.5.

Assembling bool.s produces a relocatable object file called bool .o which can be linked with the Prolog library and other modules to form an executable image file.

\subsection{The Link Phase}

The last stage consists of linking all objects resuling from Prolog sources (as explained above) with objects stemming from other sources (e.g. foreign $\mathrm{C}$ code), the GNU Prolog libraries and other objects (system or third-party libraries.) One design goal was to rely on standard compiler tools to retain only what is necessary: the linker (ld under Unix) links to an object library, from which only the required modules are taken, thereby keeping the size of the final executable down. Since a Prolog source results in a classical object file, several objects can be grouped in a library (e.g. using ar under Unix). The Prolog and FD built-in libraries are created in this way (and users can also define their own libraries). Defining a library allows the linker to extract only the object files that are necessary (i.e. those containing referenced functions/data). For this reason, GNU Prolog can generate small executables by avoiding the inclusion of most unused built-in predicates. On the other hand, the linker cannot guess which built-in predicates will be called by a meta-call. To deal with this problem, GNU Prolog provides the directive ensure_linked which guarantees that a given predicate will be linked (in fact, all it does is to create a simple reference to the predicate in the assembly file, to force the linker to pull the desired predicate in from the library). 
As previously stated, each linked object includes initialization code in which various housekeeping functions are performed. This function gets executed before any compiled Prolog code. The ELF format allows the specification of global ObjectOriented constructor code, which gets executed at the start and is collected from several object modules. We use this mechanism to initialize GNU Prolog objects.

\subsection{GNU Prolog Executable Behaviour}

From the user point of view, the behaviour of an executable produced by GNU Prolog consists of executing all intialization/1 directives. If several initialization/1 directives appear in the same file they are executed in the order of appearance. If several initialization/1 directives appear in different Prolog files (i.e. in different objects) the order in which they are executed is implementation-defined. However, on most machines the order will turn out to be the reverse of the order in which the associated files have been linked. The traditional Prolog interactive top-level interpreter is optionally linked with the rest of the executable. Should it be present, it gets executed after all the other initialization/1 directives have finished. This default behaviour is provided as a main function defined in the GNU Prolog library. So in the absence of a user-defined main function the default function is executed. Here is its definition:

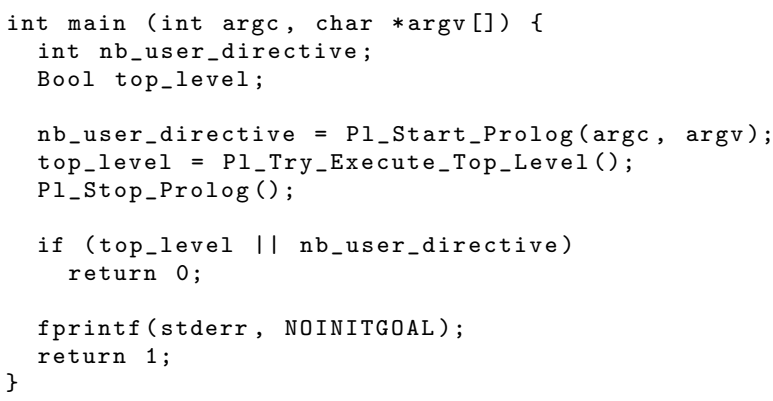

Line 5: the P1_Start_Prolog allocates all data areas (stacks, tables, etc.) and, for each registered object, in reverse order, invokes its Prolog_Object_Initializer, System_Directives and User Directives. It returns the number of directives actually executed.

Line 6: if the top-level is linked then execute it.

Line 7: free all allocated areas.

Lines 12-13: warn the user that nothing has been executed, i.e. there is no user directive and the top-level is not present in the executable.

The user can provide another main function, to customize this behaviour.

\subsection{Bootstrapping the System}

Being written in Prolog, GNU Prolog - and the p12wam compiler in particular - relies on its own availability in order to recompile itself. We now discuss some aspects of the bootstrap process.

The parts of the Prolog compiler written in Prolog 9 are expected to be compilable

9 Actually, the entire compiler is written in Prolog. 
by GNU Prolog. As a consequence, the .pl source files need to be compiled using the GNU Prolog compiler pipeline, as described in figure 1 in particular, there will have to be a .wam file for each .pl source. These files are then further compiled by the non-Prolog parts of the system (wam2ma, ma2asm, the assembler and link editor.)

Note that a running Prolog system is only needed to get to the .wam representation: from that point on, all compiler passes are implemented as $\mathrm{C}$ programs. In order to bootstrap GNU Prolog on a particular machine, one does not actually need any working Prolog compiler, as the .wam files for the pl2wam executable are provided with the source.

Another aspect worth mentioning involves quality assurance for the Prolog-toWAM compiler: a new version of the compiler should hit a fixpoint for the contents of the . wam files: the files produced by the compiler should converge to be identical to those which make up the compiler itself. The initial . wam files may be produced from the Prolog sources either by GNU Prolog or by another Prolog system. The integrity of the generated Prolog compiler is automatically verified by comparing the resulting . wam files to the ones originally provided.

\subsection{Different Code Representations}

The primary goal of GNU Prolog is to compile to native code and thus to provide standalone executables, in the sense that references within the program are statically resolved by the linker and the code is directly available for execution. Emulator-based systems appear to provide similar functionality by bundling the program bytecodes with an emulator. However, GNU Prolog has also to handle dynamic Prolog clauses. Generally speaking, GNU Prolog simply has to be able to meta-interpret. This is the case when the programmer uses the asserta or assertz built-in predicates: the clause will be stored and (meta-)interpreted. The compiler will try to do a bit better than this, in some situations: suppose, for instance, that a Prolog source file contains a :- dynamic directive for some predicate: the native code for all defined clauses is generated. At run-time, even though the predicate is dynamic, it's the native code that gets executed: this ceases being so when the clause is removed with retract/1. Clauses added at run-time will only be metainterpreted, i.e. they will have no native code counterpart. In the case of dynamic predicates, in addition to the native code, the compiler also emits a system directive which records the term associated to the clause (to be inspected using clause/2).

It can be argued that standardization efforts could have differentiated among the two situations: to have one "assert for code only" and another for "data only."

Out of respect for Prolog tradition, GNU Prolog also offers an interactive top-level. A major problem GNU Prolog has to face is the implementation of the (in)famous consult(FILE) and reconsult (FILE) predicates. Several possibilities exist in a native-code system:

1. Read FILE, assert the code and have it meta-interpreted.

2. Compile FILE to byte-codes, which will be interpreted by a WAM byte-code emulator. 
3. Compile FILE to native code and find a solution to dynamically load it into the running process.

The first solution is simple to implement but obviously not very efficient. For the time being, we settled for the second solution: we have developed a simple emulator to execute a binary representation of the code provided by pl2wam. This emulator is not optimized at all but provides a speedup of about 3 when compared to metainterpreted code.

We plan on moving to the third approach, which is becoming feasible in a portable way by resorting to native shared libraries, which can be dynamically loaded or released from the running process memory. Following this route frees us from having to use the byte-code interpreter. On the downside, the production of native code that can be dynamically loaded is a bit more demanding because the machine code has to be position-independent, which requires rewriting of the architecture-specific back-ends for ma2asm.

To summarize, GNU Prolog currently manages three kinds of code:

- interpreted code for meta-call and dynamically asserted clauses

- emulated (byte-)code for consulted predicates

- native code for statically compiled predicates

As a result, these three ways of representing and executing Prolog programs need to be integrated, which turned out to be a demanding requirement, as these models differ quite a bit. GNU Prolog has by no means the exclusivity as far as this aspect is concerned: other Prolog systems need to represent programs in more than two ways (BIM-Prolog and SICStus for instance.)

\subsection{Discussion}

This concludes the presentation of the GNU Prolog compilation scheme. Some goals or aspects of the system are comparable to other systems, for instance the native code implementation for SICStus Prolog of (Haygood 1994) or Aquarius (Van Roy and Despain 1992) which also aim at compiling Prolog to interpreter-less native code for real architectures.

With respect to the direct generation of native code as opposed to going through $\mathrm{C}$, the latter has the advantage that it is easier to set up, more portable and maintainable. The downsides include high compilation times (as a result of using a general-purpose, optimizing $\mathrm{C}$ compiler), relatively low performance when generating standard $\mathrm{C}$ code and, should one strive to improve performance by using nonstandard extensions to the $\mathrm{C}$ language, the maintenance effort of the $\mathrm{C}$ compiler itself. Our option of direct native code generation benefits from much better compilation times and potentially very high performance at runtime. The main drawback of this approach is its maintainability: new targets must be explicitly programmed and adding new cross-cutting features to the language or model requires an adaptation of all the back-ends (e.g. threads or dynamic linking.)

Native code generation is usually aimed at high performance. The potential is 
high: absolute control over hardware register usage 10 optimal tagging schemes, precise control flow, to name but a few aspects. However, in order to tap into this potential, rich intermediate representations need to be devised. Such was the option in Aquarius Prolog, which defined the BAM (Van Roy and Despain 1992), significantly different from the WAM, including several "realistic" low-level finegranularity instructions. Likewise, native code generation within SICStus Prolog led to the definition of an intermediate language, the SAM (SICStus Abstract Machine) which was translated into M68K assembler or, alternatively, further compiled into yet another low level representation (RISS) which was then mapped to a specific machine language (Sparc or MIPS). Neither survived: the Aquarius compiler remained unusably slow and native code generation was dropped from SICStus because of its maintenance requirements.

It can be argued that most efforts in designing and implementing lower-level abstract machines for Prolog were targeting RISC architectures. For instance it used to be a challenge to effectively use the fine-grained control of pipeline and instruction flow that was typical of, say, MIPS or Sparc processors. Nowadays, most available microprocessors implement a common architecture (x86 or x86-64) but specific hardware implementations have sufficiently differing pipeline structures that it becomes very difficult to optimize for any one of these. Besides, dynamic instruction reordering also makes static instruction scheduling a largely moot point.

Performance in modern architectures is heavily dependent on making good use of the memory cache hierarchies; Prolog compiler writers stand to gain a lot from making good use of cache organizations, possibly more so than what can be bought by other optimization techniques. The problem is that there is a lot of variation across systems that must be accounted for to extract optimal performance.

It turns out that the more sophisticated approaches to native code generation for Prolog have somehow vanished in the long run, while GNU Prolog remains upto-date and has been ported to several low-level architectures. We feel we have achieved a good balance between simplicity, maintainability and performance. To pursue performance gains without sacrificing simplicity we are investigating a replacement for the MA level in GNU Prolog: we are presently evaluating tools such as LLVM (Lattner and Adve 2004) which can be thought of as a typed, machineindependent assembly language.

\section{Finite-Domain Constraints}

The main extension built on top of wamcc was arguably clp(FD), which added constraint solving over Finite Domains (FD). GNU Prolog compiles FD constraints in a way similar to its predecessor clp(FD), the approach being described in (Codognet and Diaz 1996, Diaz and Codognet 1993). It is based on a so-called "RISC approach" which consists of translating, at compile-time, all complex user-constraints (e.g. disequations, linear equations or inequations) into simple, primitive constraints

10 Being able to use hardware registers favours a register-based abstract machine such as the WAM, as opposed to other approaches. 
(the FD constraint system) which operate at a lower level and which really embody the propagation mechanism for constraint solving. We shall first present the basic ideas of the FD constraint system and then detail the extensions to this framework implemented in GNU Prolog.

The FD Constraint System was originally proposed by Pascal Van Hentenryck in the concurrent constraint setting (Van Hentenryck et al. 1994), and an efficient implementation in the clp(FD) system is described in (Codognet and Diaz 1996 Diaz and Codognet 1993). FD is based on a single primitive constraint with which complex constraints are encoded: for example, constraints such as $X=Y$ or $X \leq$ $2 Y$ are defined by means of FD constraints, instead of having to be explicitly built into the theory. Each constraint is made of a set of propagation rules describing how the domain of each variable is related to the domain of the other variables, i.e. rules for describing node and arc consistency propagation (see for instance (Tsang 1993) for more details on CSPs and consistency algorithms.)

A constraint is a formula of the form $X$ in $r$ where $X$ is a variable and $r$ is a range. A range in FD is a non empty finite set of natural numbers. Intuitively, a constraint $X$ in $r$ enforces that $X$ belongs to the range denoted by $r$. Such a range can be a constant range (e.g. 1..10) or an indexical range, when it contains one or more of the following:

- $\operatorname{dom}(Y)$ which represents the whole current domain of $Y$;

- $\min (Y)$ which represents the minimal value of the current domain of $Y$;

- $\max (Y)$ which represents the maximal value of the current domain of $Y$.

- $\operatorname{val}(Y)$ which represents the final value $Y$ (i.e. the domain of $Y$ has been reduced to a singleton). A constraint involving such an indexical is delayed until $Y$ is determined.

Obviously, when $Y$ is instantiated, all indexicals evaluate to its value. When an $X$ in $r$ constraint uses an indexical term depending on another variable $Y$ it becomes store-sensitive and must be checked each time the domain of $Y$ is updated. This is how consistency checking and domain reduction is achieved.

Complex constraints such as linear equations or inequations, as well as symbolic constraints can be defined in terms of FD constraints, see (Codognet and Diaz 1996) for more details. For instance, the constraint $X \leq Y$, is translated as follows ${ }^{11}$

$$
\mathrm{X} \leq \mathrm{Y} \equiv \mathrm{X} \text { in } 0 . . \max (\mathrm{Y}) \wedge \mathrm{Y} \text { in } \min (\mathrm{X}) . . \infty
$$

Notice that this translation also has an operational flavor and specifies, for a given n-ary constraint, how the domain of a variable may be updated in terms of the other variables. For example, consider the FD constraint $\mathrm{X}$ in $0 . . \max (\mathrm{Y})$ : whenever the largest value of the domain of $\mathrm{Y}$ changes (i.e. decreases), the domain of $\mathrm{X}$ must be reduced. If, on the other hand, the domain of $\mathrm{Y}$ changes but its largest value remains the same, then the domain of $\mathrm{X}$ does not change. One can therefore consider those primitive $\mathrm{X}$ in $\mathrm{r}$ constraints as a low-level language in which to express the propagation scheme. Indeed, it is possible to express in the constraint definition

${ }^{11}$ In this discussion, we're not using the GNU Prolog concrete syntax for constraint goals. 
(i.e. the translation of a high-level user constraint into a set of primitive constraints) the propagation scheme chosen to solve the constraint: forward-checking, full or partial look-ahead, according to the use of dom or min/max indexical terms.

\subsection{The Constraint Definition Language}

For GNU Prolog, we designed a specific language to define FD constraints which is both flexible and powerful. The basic $X$ in $r$ is sufficient to define simple arithmetic constraints but too restrictive to handle constraints like $\min (X, Y)=Z$ or reified constraints, both of which need some form of delay mechanism. Another limitation is that it is not possible to explicitly indicate the triggers for a particular propagator: these are deduced from the indexical used in the $X$ in $r$ primitives. The GNU Prolog constraint definition language, FD, has then been designed to allow the user to define complex constraints and proposes various constructs to overcome these limitations. FD programs are compiled into $\mathrm{C}$ by the $\mathrm{fd2c}$ translator. The resulting $\mathrm{C}$ program is then compiled and the object fits into the compilation scheme shown in Figure 1. We present the main features of the constraint definition language by means of a few examples.

\subsubsection{Arithmetic Constraint Definition}

Consider a constraint $X+C=Y$ ( $X$ and $Y$ are FD variables, $C$ is an integer):

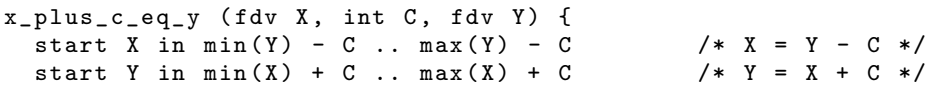

Constraints are defined in a C-like syntax. The head declares the name of the constraint (x_plus_c_eq_y) and for each argument its type (fdv, int) and its name. The keyword start activates an $X$ in $r$ primitive. The first states that the bounds of $X$ must be between $\min (Y)-C$ and $\max (Y)-C$. Similarly, the second rule indicates how to update $Y$ from $X$.

Take a more complex example, which defines $\min (X, A)=Z$ (where $X$ and $Z$ are FD variables and $A$ an integer):

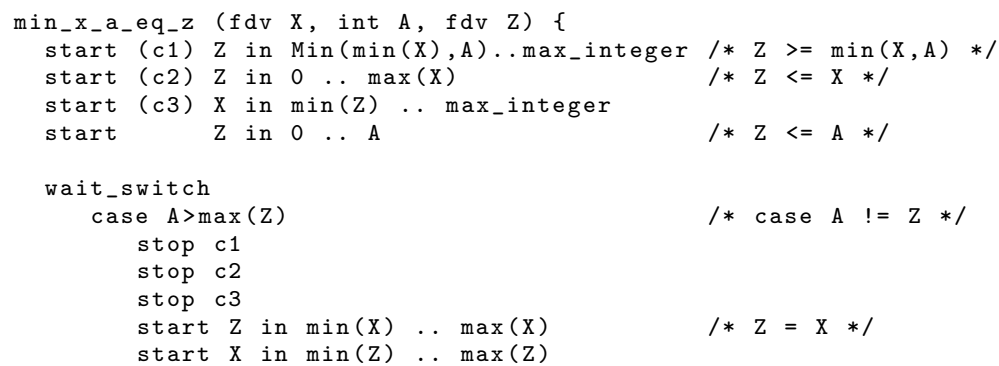

The first $X$ in $r$ constraint uses a $\mathrm{C}$ macro Min to compute the minimum of $\min (X)$ and $A$. The keyword max_integer represents the greatest integer that an FD variable can take. Note the use of the wait_switch instruction to enforce $X=Z$ (and to stop the constraints $\mathrm{c} 1, \mathrm{c} 2, \mathrm{c} 3$ ) as soon as the case $A \neq Z$ is detected. 


\subsubsection{Reified Constraint Definition}

The facility offered by the language to delay the activation of an $X$ in $r$ constraint makes it possible to define reified constraints: the basic idea of a reified constraint is to consider the truth value of a constraint as a first-class object, which is given the form ("reified") of a boolean value. This allows the user to make assumptions about the satisfiability of constraints in a given store in order to conditionally require that other constraints be met. It is feasible to use this mechanism, for instance, to define disjunctive constraints, which can be very useful to model complex problems.

The following example illustrates how to define $X=C \Leftrightarrow B$ where $X$ is an FD variable, $C$ an integer and $B$ a boolean variable (i.e. an FD variable whose domain is $0 . .1$ ) which captures the truth value of the constraint $X=C$. The definition below waits until either the truth of $X=C$ or the value of $B$ is known:

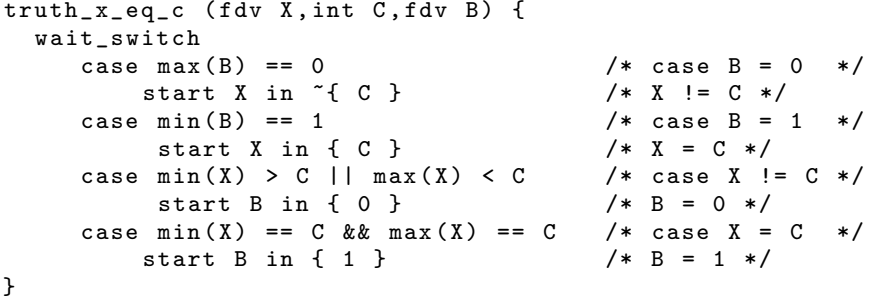

Each constraint results in a $\mathrm{C}$ function returning a boolean depending on the outcome of the addition of the constraint to the store. The link between Prolog and a constraint is done by the Prolog built-in predicate $f d_{-} t e l l / 1$. For instance, to use the previous constraint one could define the following predicate:

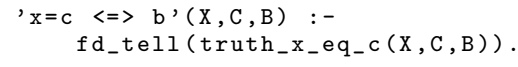

\subsubsection{Global Constraints}

Global constraints allow the user to specify patterns that are frequently encountered in problems. A global constraint can often be decomposed into simple (local) constraints. However the pruning obtained with such a decomposition is less efficient than that provided by specialized propagation algorithms. The GNU Prolog constraint language is not expressive enough to describe any filtering procedure which has to be written in C. An API is provided to the $\mathrm{C}$ programmer for handling FD variables, ranges, etc. To simplify the interface between Prolog and $\mathrm{C}$ for constraints, the FD language offers the following facilities:

- It handles lists of FD variables and/or integers (types $1 \_f d v$ and $1_{-}$int). At run-time, a corresponding Prolog list is expected and it is passed to the $\mathrm{C}$ code as a $\mathrm{C}$ array (of pointers to $\mathrm{FD}$ variables or of integers).

- It implicitly wakes up the constraints suspended on indexicals occurring in the lists (but the user can define another triggering strategy).

- It can invoke a user-defined $\mathrm{C}$ function to compute a range in a $X$ in $r$ primitive or outside any primitive. 
Consider the element $(I, L, V)$ constraint which says that the $I$ th element of integer list $L$ is equal to $V$ ( $I$ and $V$ are FD variables). It is defined as follows:

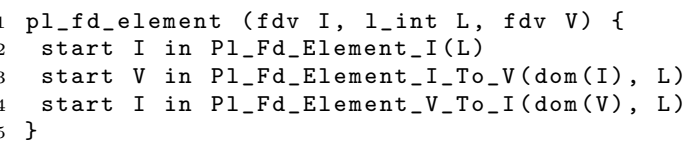

The first constraint is executed only once to set the initial domain of $i$ to 1 ..length $(L)$. The second constraint is woken up each time the domain of $I$ is modified, in order to compute the new domain of $V$. To this effect, the $\mathrm{C}$ function P1_Fd_Element_I_To_V is called. It mostly iterates over each value $j$ from the domain of $I$, accumulating the $j^{\text {th }}$ element of the list $L$. The simplified C code of this function looks like:

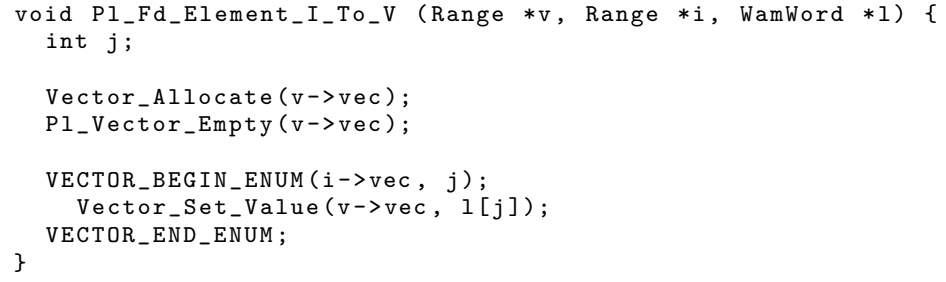

Line 1: The function accepts i (the current domain of the variable $I$ ) and 1 [] (the array associated to the list of integers $L$ ) and computes $\mathrm{v}$, the new domain of the variable $V$ (this will be stored in the first argument of the function). Note: the tell of Vinv is not done here but by the $X$ in $r$ primitive at the return of the function).

Line 4-5: A bit-vector is allocated and cleared (v).

Line 7-9: Using $C$ macros, the values of the domain of $I$ are scanned. For each value $j$, the corresponding element in $L(\mathrm{I}[\mathrm{j}])$ is accumulated in v.

Conversely, the third constraint is triggered each time the domain of $V$ is modified to compute the new domain of $I$. To this end, the $\mathrm{C}$ function P1_Fd_Element_V_To_I iterates over all values in $L$ which are also present in $V$, accumulating their indexes.

GNU Prolog offers a variety of high-level constraints in the built-in library. Lowlevel definitions of constraints as illustrated here are, however, open to the expert programmer who needs to customize or enrich the constraint solver for some practical application. At the moment the ultimate customization is achieved by writing $\mathrm{C}$ code. This smoothly integrates into the native-compilation scheme adopted by GNU Prolog. We do plan, however, to extend the expressive power of the language to be able to describe some global constraints without adding any $\mathrm{C}$ code.

\subsection{Integrating Constraints into the WAM}

We here recall the main points of the integration of FD constraints into the WAM (see (Codognet and Diaz 1996) for more detailed information). To understand the necessary data structures one needs to study the basic consistency procedure. When a $X$ in $r$ constraint is added, the range $r$ is evaluated and the domain of $X$ is updated accordingly (the new domain of $X$ being the intersection between its current domain and $r$ ). Once this is done, propagation may occur: every constraint on $Y$ which depends on $X$ (e.g. Y in $\min (X)+10 \ldots \max (X)+10)$ needs to be reevaluated. 
Doing so will potentially modify $Y$ and all constraints depending on $Y$ also need to be reconsidered. The process finishes either when a failure occurs (the new domain of a variable is empty) or when a fix-point is reached (no more variables are modified). In case of failure, Prolog backtracking occurs. It is then important to be able to undo all modifications that have been done on the FD data structures.

Adding constraints over finite domains to the GNU Prolog WAM required the introduction of a new term type (FD Variable, with the FDV tag) which, besides contributing to tag space depletion, needs to be distinct from the regular REF term. An FDV term has 2 distinct parts:

- Its domain: the set of allowable values, represented as the extrema of the containing interval or as discrete individual values, encoded as a bitmap, possibly multi-word. Using a bitmap greatly speeds up computation on sparse domains.

- The dependencies: the set of constraints which depend on the variable, i.e. those which need to be recomputed each time the variable is modified. In order to optimize the triggering of these constraints, several distinct chains are maintained (e.g. it is useless to re-execute a constraint depending on $\min (X)$ when only $\max (X)$ is changed).

Classically, a value-trail mechanism is used to save an FD variable before its modification (domain and/or dependencies). On backtracking, trailed values are used to restore the FD variable. In order to avoid unnecessary trailings (for each FD variable, at most one trailing is necessary per choice-point) a timestamp technique is used: a sequential integer is used to number each choice-point and an FD variable records the choice-point number associated to its last trailing. This is important since FD variables are refined step by step by the propagation algorithms which potentially compute several intermediate domains before reaching the fix-point.

The two parts of an FD variable (domain and dependencies) are generally not modified at the same time during the execution of a constraint program. The dependency chains are created and updated when the constraints are installed, typically at the start of program execution, whereas the domains are more intensely modified later on, for instance during the labeling phase which tries to find a solution through backtracking. For this purpose, each part of an FD variable (domain and dependencies) maintains its own independent timestamp. In particular, when doing labeling, we only trail the domain of the FD variables.

The other important data structure is the constraint frame, which stores the information needed for constraint (re)evaluation. For an $X$ in $r$ primitive we need:

- A pointer to the constrained variable $X$.

- The address of the $\mathrm{C}$ function evaluating the range $r$ (this is produced by $\mathrm{fd} 2 \mathrm{c}$ from the definition written in the constraint definition language).

- A pointer to the environment in which the function evaluating $r$ executes: basically the function parameters, built by the constraint installation code.

We chose a dedicated stack in which to store all these data structures, called the constraint stack. As for other Prolog data strucutures, the stack is used in backtracking: the top of the constraint stack is saved in choice-points and restored when 
backtracking occurs. This was not the case in clp(FD) where all FD data structures were located in the heap. In the tests we conducted, the performance impact of having a constraint stack was negligible on programs which did not use FD.

From the above propagation algorithm it appears that the evaluation of a constraint leads to the reevaluation of other constraints. Theoretically, the order in which constraints are woken up is not relevant (since the process stops when the fix-point is reached). The easiest way to implement this consists of a depth-first evaluation (recursively calling each constraint depending on the variable which has just been updated). However, this blind recursive descent is not efficient in practice and misses some important optimizations. It is thus better to explicitly handle a queue of constraints. A first optimization consists of considering a queue of variables instead of a queue of constraints. When a constraint needs to be reevaluated, it is as consequence of the modification of some FD variable. It is easier to record just this modified variable (a pointer) than to copy in the queue all depending constraints. In GNU Prolog we go even further: the queue is not separately represented: instead, all FD variables present in the queue are linked together. To this end, an FD variable (see above) includes a third part which is devoted to the queue. It consists of:

- A link to the next enqueued variable (linked-list).

- A mask describing which dependencies need to be reconsidered (to avoid useless reevaluations).

- A time-stamp to know whether a variable is already present in the queue. There is a general counter which is incremented each time the (above) propagation procedure is run. When a variable is modified, if its time-stamp is different from the counter then the variable is not yet in the queue (it is then linked), otherwise only its mask of dependencies is updated.

Note that our choice for the representation of the queue associated with the timestamp technique described above results in an optimization: the constraints depending on one variable are only present once in the queue. On a set of benchmarks, this optimization saves an average $17 \%$ of the execution time (it is particularly effective on arithmetic constraints) with no overhead. Detecting this case with a separate queue would be much more time-consuming.

Another optimization which works well in practice for many constraints is that an $X$ in $r$ primitive does not need to be evaluated if $X$ has been instantiated before the start of the propagation procedure. This can be detected reusing the same counter described above. On some examples this optimizations saves up to $72 \%$ of the execution time (in particular when many disequalities are involved).

We have shown that GNU Prolog smoothly integrates an efficient FD solver, proposing a simple yet powerful language in which to describe high-level constraints and propagators. Those constraints are compiled down to $\mathrm{C}$ code, which in turn is integrated into the GNU Prolog executable build flow of figure 1. More constraints can then easily be added thanks to the description language and if needed with the help of dedicated user-defined C functions. The compilation of high-level constraints is based on a limited set of primitives which are well optimized. These optimizations 
are "general" (vs. "ad-hoc optimizations" of black-box solvers). So all high-level constraints can benefit from them.

\section{GNU Prolog and the Prolog Standard}

From the outset, GNU Prolog has aimed to comply with common practice in Prolog implementations, while retaining its characteristic architectural organization: to fit into a regular native code compiler system, in which executables are produced by linking object modules.

GNU Prolog was developed at the same time as the ISO Core 1 standard (ISOPart1 1995), which led us to take the standard proposal into account from the outset. GNU Prolog therefore became the first Prolog system to closely comply with the ISO standard. This meant supplying not only the standard built-in predicates but also the related error behaviours (e.g. exceptions), the logical database update view for dynamic predicates, meta-calls (the ISO standard requires a term to be transformed into a goal before execution), directives, etc. ${ }^{12}$

We took compatibility one step further by providing a classical Prolog top-level interpreter, with all the expected facilities operational, including goal execution, source display (the listing/0 predicate), a trace-mode 4-port debugger, program consult and reconsult, Prolog state manipulation operations (character classification, operator definitions, etc.) We do think that a top-level interpreter is a primitive form of Integrated Development Environment (IDE): it makes historic sense, but it would be better to integrate stripped-down compiler-like tools into a graphical IDE such as Eclipse, NetBeans or Xcode by means of a plug-in.

It can be argued that the DEC-10 Prolog compiler was influential in many ways and some aspects of its design persist in today's Prolog systems. Its operating environment set a model which would be emulated by most Prolog systems which came thereafter: the interactive top-level with a "workspace" concept, which contains the whole of the program, all seamlessly integrated, regardless of the representation used for Prolog code: clausal form suitable for a meta-interpreter, lower-level instructions adapted to a byte-code interpreter or even executable native code.

This model holds, among others, for DEC-10 Prolog, C-Prolog, Quintus, SICStus, MU-Prolog and Nu-Prolog, YAP, XSB, SWI, Ciao, BinProlog, ECLiPSe and BProlog. With such a heritage, GNU Prolog was almost compelled to follow suit and establish itself around the concept of a top-level interpreter managing goals executed in the context of a dynamically-adjustable workspace, comprising all the Prolog modules and equipped with a 4-port debugger, familiar to Prolog programmers, although not strictly part of the language.

The functional enrichment of Prolog systems, and in particular those features that stem from the language's meta-programming capabilities, went forth basically

12 It is worth noting that GNU Prolog has kept up with the proposed revisions to the standard, including features such as call/N, conditional compilation directives and evaluable functors, among others. 
unaware of the distinction between "compile time" and "execution time" environments. This mingling is such that executing one query may influence a subsequent consult operation, in ways that may completely alter its semantics (for instance, operator definitions.)

For a system like GNU Prolog, which does independent static compilation to native code and clearly separates the compilation from the execution environments, providing a top-level interpreter similar to other systems was a challenge which required a large development effort. This requirement prompted the addition of a bytecode emulator to GNU Prolog, similar to what is done in other Prolog implementations, to provide a not-too-inefficient means of implementing the culturallyaccepted development cycle for Prolog programs: edit/reconsult/run. The grief over compile-time vs. runtime environments is not confined to GNU Prolog though: this is a prominent issue in all systems that do static analysis or program transformation, such as mode or type analyzers or even simple pretty-printers.

We feel that the ISO standard missed a good opportunity to disentangle this situation and separate compile from execution environments. In particular:

1. The :- initialization directive was meant for an interpreted environment, where one expects it to have an immediate effect on the rest of the program, whether it is simply being compiled or actually being loaded. The semantics of this directive are unclear when the driving goal is, for instance, something like consult ( $[f 1, f 2]$ ), in which the initialization directives from $f 1$ may influence the loading of $\mathrm{f} 2$. A possible way around this issue is to separate the execution of the initialization directives from the loading of the modules: GNU Prolog only executes the initializers once all modules have been loaded. The execution order is, in terms of the ISO standard, "implementation dependent."

2. Another ISO directive which causes grief is multifile/1: one problem is the order in which the multiple batches of clauses get collected. This is not an issue in an interpreted environment, in which the loading is explicitly controlled by the programmer whereas in a statically compiled set of Prolog files the order is largely unpredictable, because it is left to the linker's criteria.

3. One unfortunate feature of the Prolog language, legacy of the interpreter tradition, is the lack of distinction between code and data-only (database) dynamic predicates. The ISO standard missed the opportunity to clearly distinguish between these two traditional uses for dynamic predicates: persistent data and dynamic code manipulation.

These were but a few of the difficulties which hit us when developing GNU Prolog; nevertheless, we strived to provide a fair rendering of an expected set of built-in predicates. The "standard" Prolog library is nowhere near as complex as that of other languages so the extent of this requirement is limited. We also behave conservatively w.r.t. extra-logical aspects of Prolog, such as the handling of directives. 


\section{Conclusions and Directions for Future Work}

We have presented the most significant aspects of the implementation of GNU Prolog for which the key issues were simplicity, extensibility and maintainability without sacrificing performance. This led us to the native-code generation approach which has been described in this article. We applied the same requirements for the design and the development of the finite domain constraint solver. One might say that, overall, the GNU Prolog experience has been successful. The GNU Prolog "family", which includes wamcc and $c l p(F D)$ has been used in teaching and as the basis of several extensions, most notably by other research teams: this fulfills one of our design goals which was to establish a system sufficiently simple for it to be easily extended by other people.

Several architectural ports and some extensions have been provided by the user community, as acknowledged in the GNU Prolog distributed documentation. Modified versions of GNU Prolog have been used for prototyping systems, featuring module systems, threads, attributed variables, $\operatorname{CLP}(\mathcal{R})$, RDBMS integration, Java interfaces, a MacOSX IDE, to name but a few. The community-supplied extensions are referenced on the main site at http://www.gprolog.org/\#contribs.

In what concerns dissemination, the GNU Prolog distribution had been downloaded well over 100000 times from the development FTP site, over a period of four years. We no longer keep statistics, as GNU Prolog is part of several Linux distributions and there is no way to account for downloads from the main GNU FTP site nor from other mirrors.

Performance-wise, GNU Prolog scores honorably, barely below YAP (da Silva and Santos Costa 2006) which is continually being tuned for performance. We compared GNU Prolog 1.4.0 and YAP 5.1.3 on 64-bit Linux. On the average, YAP is faster by factor of 1.3 with peaks up to 2. However, on some benchmarks GNU Prolog can outperform YAP by a factor of up to 1.4. With respect to wamcc we clearly gained in usability, as a consequence of the more realistic compile times. GNU Prolog is currently being worked on in various directions, including:

- Modules: GNU Prolog initially did not implement any module system, staying within the bounds of ISO Prolog Core 1, awaiting the ISO Modules specification. Reaching a consensus on modules took a long time and the resulting specification is still not very satisfactory. We initially opted for the implementation of a cleaner alternative mechanism, Contextual Logic Programming (Abreu and Diaz 2003). Nevertheless, as there is a clear need for an interoperable module system, we are finishing a minimal-functionality module system as part of the Prolog Commons initiative, which brings GNU Prolog at par with the other implementations.

- Other ISO Prolog Features: ISO compliance has been foremost in the design and implementation of GNU Prolog; the work being carried out by the ISO standization committee is being actively followed. For instance, one aspect that needs to be accounted for is the handling of Unicode characters.

- Attributed Variables: even though GNU Prolog has a very efficient, convenient and easily extensible FD constraint solver, it makes sense to include other 
constraint domains. Attributed variables are a mechanism which can be used to effectively implement constraints and propagation over other domains.

- Tabling: using tabling allows one to write programs which are more expressive because the system takes care of memoizing for us. More programs terminate which would otherwise loop and this can be a very effective programming device. This extension to Prolog was introduced in XSB (Rao et al. 1997) and has since been included in other systems, namely YAP (Rocha et al. 2000, Rocha et al. 2005) and B-Prolog (Zhou et al. 2008).

- A Garbage Collector: GNU Prolog has gotten by without GC. While reasonable for short-lived processes ${ }^{13}$ it is a limiting factor for larger executions.

- Improved Compiler: the GNU Prolog Prolog-to-WAM compiler is rather simple. This is an obvious area for improvements.

- Compilation Pipeline: because it is made up of a succession of filters, GNU Prolog is amenable to the substitution of some of these: we are presently working on a few, for instance one which manages EAM-style executions from the WAM code. A longer-term goal is to rework the GNU Prolog back-end and improve its integration into an existing compiler scheme: LLVM (Lattner and Adve 2004) is an interesting target, as it is essentially a machine-independent, typed assembly language which could take over the MA and Assembly language steps.

Acknowledgements: The authors wish to acknowledge the anonymous reviewers who read early versions of this text and whose criticisms contributed to significantly improve its form and substance.

\section{References}

Abreu, S. And Diaz, D. 2003. Objective: In minimum context. In ICLP, C. Palamidessi, Ed. Lecture Notes in Computer Science, vol. 2916. Springer, 128-147.

AїT-KACI, H. 1991. Warren's Abstract Machine: A Tutorial Reconstruction. MIT Press. André, P. And Abreu, S. 2010. Casting the WAM as an EAM. In Proceedings of CICLOPS-WLPE 2010, G. Vidal and N.-F. Zhou, Eds.

Carlsson, M. 1990. Design and Implementation of an Or-Parallel Prolog Engine. Ph.D. thesis, SICS.

Chikayama, T., Fujise, T., And Sekita, D. 1994. A portable and efficient implementation of kl1. In PLILP, M. V. Hermenegildo and J. Penjam, Eds. Lecture Notes in Computer Science, vol. 844. Springer, 25-39.

Codognet, P. And Diaz, D. 1995. Wamcc: Compiling prolog to c. In ICLP. 317-331.

Codognet, P. And Diaz, D. 1996. Compiling constraints in clp(fd). Journal of Logic Programming 27, 3, 185-226.

DA Silva, A. F. And Santos Costa, V. 2006. The design of the yap compiler: An optimizing compiler for logic programming languages. J. UCS 12, 7, 764-787.

Diaz, D. And Codognet, P. 1993. A minimal extension of the wam for $\mathrm{clp}(\mathrm{fd})$. In ICLP. $774-790$.

13 GNU Prolog is very suitable for building small executables, with short load times: Unix systems share code sections and this means code lingers even when no processes are using it. This is normally not the case with bytecodes kept in writeable, non-shared memory. 
Ferreira, M. AND DAmas, L. 1999. Multiple specialization of wam code. In PADL, G. Gupta, Ed. Lecture Notes in Computer Science, vol. 1551. Springer, 243-258.

Gudeman, D., De Bosschere, K., And Debray, S. 1992. jc: An Efficient and Portable Sequential Implementation of Janus. In Joint International Conference and Symposium on Logic Programming. MIT Press, Washington, DC.

Hausman, B. 1993. Turbo erlang: Approaching the speed of c. In ICLP-Workshops on Implementation of Logic Programming Systems. 119-135.

HAYGOOD, R. C. 1994. Native code compilation in sicstus prolog. In In Proceedings of the Eleventh International Conference on Logic Programming. MIT Press, 190-204.

ISO-Part1 1995. Information technology - Programming languages - Prolog - Par t 1: General Core. ISO/IEC 13211-1.

Jaffar, J. AND LAssez, J.-L. 1987. Constraint logic programming. In POPL. 111-119.

Jones, S. L. P., Nordin, T., And Oliva, D. 1997. C--: A Portable Assembly Language. In Implementation of Functional Languages, C. Clack, K. Hammond, and A. J. T. Davie, Eds. Lecture Notes in Computer Science, vol. 1467. Springer, 1-19.

Lattner, C. AND Adve, V. 2004. LLVM: a compilation framework for lifelong program analysis transformation. In Code Generation and Optimization, 2004. CGO 2004. International Symposium on. $75-86$.

Rao, P., Sagonas, K., Swift, T., Warren, D., and Freire, J. 1997. XSB: A system for efficiently computing well-founded semantics. In Proceedings of the 4th International Conference on Logic Programming and Non-Monotonic Reasoning (LPNMR'97). Vol. 1265. $2-17$.

Rocha, R., Silva, F., And Santos Costa, V. 2000. YapTab: A tabling engine designed to support parallelism. In Conference on Tabulation in Parsing and Deduction. 77-87.

Rocha, R., Silva, F. M. A., And Costa, V. S. 2005. On applying or-parallelism and tabling to logic programs. TPLP 5, 1-2, 161-205.

Tsang, E. 1993. Foundations of Constraint Satisfaction. Academic Press.

VAn Hentenryck, P. 1989. Parallel constraint satisfaction in logic programming: Preliminary results of chip within pepsys. In ICLP. 165-180.

Van Hentenryck, P., Saraswat, V. A., And Deville, Y. 1994. Design, implementation, and evaluation of the constraint language $\mathrm{cc}(\mathrm{fd})$. In Constraint Programming, A. Podelski, Ed. Lecture Notes in Computer Science, vol. 910. Springer, 293-316.

Van Roy, P. And Despain, A. M. 1992. High-performance logic programming with the aquarius prolog compiler. IEEE Computer 25, 1, 54-68.

Warren, D. H. D. 1983. An Abstract Prolog Instruction Set. Technical Note 309, SRI International.

Zhou, N.-F., Sato, T., And Shen, Y.-D. 2008. Linear tabling strategies and optimizations. TPLP 8, 1, 81-109. 